\title{
CARACTERIZACIÓN GENÉTICA DE EJEMPLARES DE PAICHE Arapaima gigas (Cuvier, 1829) UTILIZADOS EN EL REPOBLAMIENTO DEL LAGO IMIRIA (CUENCA DEL RÍO UCAYALI)
}

\author{
Carmen GARCIA-DAVILA ${ }^{1,3}$, Diana CASTRO-RUIZ ${ }^{1,3}$, Werner CHOTA-MACUYAMA ${ }^{1,3}$, Claudia BIFFI ${ }^{1}$, Sonia \\ DEZA $^{1}$, Roger BAZAN ${ }^{1}$, Juan GARCIA ${ }^{1}$, Mariano REBAZA ${ }^{1}$, Carmela REBAZA ${ }^{1}$, Carlos CHAVEZ ${ }^{1}$, Fred CHU- \\ $\mathrm{KOO}^{1,3}$; Fabrice DUPONCHELLE ${ }^{2,3}$, Jesús NUÑEZ ${ }^{2,3}$, Jean-François RENNO $^{2,3}$
}

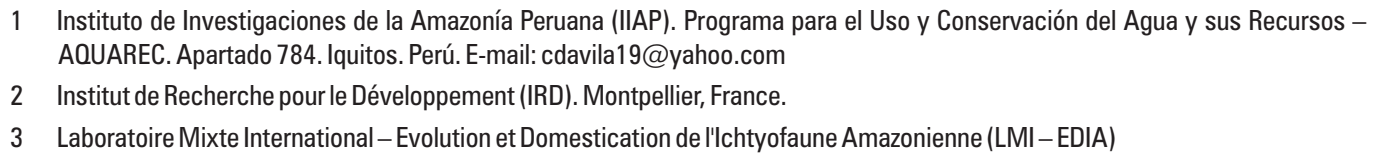

\section{RESUMEN}

El paiche Arapaima gigas, es una de las especies de peces amazónicos con fuerte presión de pesca y subsecuente disminución de sus poblaciones naturales, por lo que es nominado como especie vulnerable en el apéndice II de CITES. En ese sentido el presente estudio tuvo como objetivo contribuir al manejo y conservación de esta especie a través de la caracterización genética de 400 ejemplares de paiche utilizados en el programa de repoblamiento del lago Imiría. Los resultados de la caracterización de las seis regiones microsatélites muestran un total de 68 genotipos, con una distribución desproporcional de los mismos entre los ejemplares estudiados: dos genotipos son compartidos entre solamente 153 individuos (82 AW y 71 AX respectivamente), mostrando una gran homogeneidad genética; mientras que 36 genotipos fueron genotipos únicos, es decir cada genotipo estuvo presente en un solo individuo. Una análisis factorial de correspondencia (AFC) muestra dos principales agrupaciones entre los ejemplares estudiados, la primera conformada por 61 perfiles genéticos que reúnen 393 ejemplares $(98.25 \%)$, y la segunda por seis genotipos con solo siete ejemplares $(1.75 \%)$. Los valores de heterocigosidad esperada (He) variaron de 0 en el locus Agi13 a 0.925 en el locus Agi12. En tanto que los valores de heterocigosidad observada (Ho) variaron de 0.002 en el locus Agi 4 a 0.634 en el locus Agi10. Este elevado nivel de homogeneidad encontrado entre los ejemplares de paiche es probablemente un indicativo del alto nivel de parentesco entre estos individuos, es decir que probablemente descienden de un grupo reducido de reproductores provenientes de la piscicultura. Por lo que se recomienda que las futuras introducciones de especimenes de paiche en el lago Imiria consideren antes el genotipado de los ejemplares a utilizar en los programas de repoblamiento para asegurar la introducción de nuevos genotipos que permitan aumentar la diversidad genética del paiche en esta área.

PALABRAS CLAVE: Marcadores moleculares, microsatélites, Amazonía peruana

\section{GENETIC CHARACTERIZATION INDIVIDUALS OF PAICHEArapaima gigas (CUVIER, 1829) USED IN THE REPOPULATION OF THE LAKE IMIRIA (UCAYALI BASIN)}

\begin{abstract}
The paiche Arapaima gigas, is a species of Amazonian fish with heavy fishing pressure and subsequent decrease in natural populations, so it is nominated as a threatened species in Appendix II of CITES. In this sense, the present study aimed to contribute to the management and conservation of this species through genetic characterization paiche 400 copies of the program used in repopulate the lake Imiria. The results of the characterization of six microsatellite regions show a total of 68 genotypes, with a disproportionate distribution of the same among the specimens studied: AW and AX genotype present in 153 individuals (82 and 71 respectively), showing great genetic homogeneity; while 36 genotypes were present in one individual. FCA results show two main groupings found between genotypes, the first consisting of 61 genetic profiles that meet 393 specimens $(98.25 \%)$, and the second of six genotypes with only seven specimens (1.75\%). This high level of homogeneity between specimens found paiche is probably indicative of the high level of relatedness between these individuals, is probably descended from a small group of players. It is recommended that future introductions of specimens of paiche in the lake Imiria consider before genotyping of specimens to be used in repopulation programs to ensure the introduction of new genotypes that increase genetic diversity of paiche in this area.
\end{abstract}

KEYWORDS: Molecular markers, microsatellites, Peruvian Amazon 


\section{INTRODUCCIÓN}

La economía en la Amazonía peruana se sustenta en el aprovechamiento de sus recursos naturales, principalmente relacionados con la actividad forestal, agrícola, pesquera y en los últimos años también con la actividad minera. Estas actividades extractivas cuando son desarrolladas sin tener en cuenta la sostenibilidad social y ecológica de las mismas, pueden ocasionar serios deterioros y contaminación al medio ambiente. Esto trae como consecuencia una drástica disminución en las poblaciones naturales de animales y plantas silvestres que en ellas habitan.

En las Regiones de Loreto y Ucayali existe una fuerte demanda de carne de pescado, especialmente paiche Arapaima gigas (Cuvier, 1829), la cual hasta hace poco provenía exclusivamente de la extracción en ambientes naturales. Esta especie es uno de los peces de agua dulce más grande de Sudamérica, llegando a crecer hasta tres metros y pesar más de $200 \mathrm{~kg}$ (Nelson, 1994). Tradicionalmente, Arapaima forma parte de la dieta de una porción significativa de pobladores amazónicos, especialmente de aquellos que viven cerca de los grandes lagos de la planicie de inundación. La explotación comercial del paiche comenzó a principios del siglo 18 , debido principalmente a la calidad de su carne $(36.5 \%$ de proteína y carencia de espinas intermusculares), por lo cual en el Brasil es considerado como sustituto para el bacalao (Gadus morhua) seco salado. En el siglo XIX y XX más de 3,000 toneladas de paiche fueron exportadas por año a partir de la Amazonía brasileña (Veríssimo, 1895; Menezes, 1951), lo que dio lugar a la muerte de aproximadamente 150,000 ejemplares de paiche por año. Según los registros pesqueros hasta los años 60 el paiche era abundante cerca de las grandes ciudades de la Amazonía continental, pero a partir de los años $70 \mathrm{y}$ los 80, el paiche se convirtió en una especie muy escasa y comercialmente extinta cerca de las grandes ciudades amazónicas (Goulding, 1980). Formando parte de las especies vulnerables del CITES II desde 1975.

El lago Imiría ubicado al sur este de la ciudad de Pucallpa (distrito de Masisea. Provincia de Coronel Portillo) no fue ajena a esta presión de pesca, habiendo constituido uno de los principales ambientes de donde se extraía este recurso en la Amazonía peruana, lo cual llevo a una disminución drástica de las poblaciones naturales de esta especie en esta área. Por lo que el IIAP $y$ el IRD en el marco de un trabajo interinstitucional con La Dirección de la Producción de Ucayali (DIREPRO - Ucayali) y el Gobierno Regional de Ucayali (GOREU) en el 2009 se propusieron desarrollar un programa de repoblamiento con pre adultos de paiche de tres años de edad en el lago Imiría. El propósito de este proyecto fue recuperar las poblaciones de este importante recurso en el área, a fin de que en un periodo de tres años (duración estimada del proyecto) estos animales se reproduzcan y las poblaciones ribereñas puedan beneficiarse posteriormente de una pesca sostenida de este recurso. Además, este proyecto busco incrementar y mejorar el conocimiento sobre la biología de estos peces con relación a reproducción, crecimiento, dispersión, comportamiento, variabilidad genética, de tal manera que se pueda establecer una línea base para el manejo y conservación del paiche en estos ambientes. En ese sentido el presente estudio tuvo como objetivo la caracterización genética de los ejemplares de paiche utilizados en el programa de repoblamiento. Esto con la idea de monitorear la evolución de la variabilidad genética de la población en el tiempo y determinar su grado de adaptación a estos ambientes.

\section{MATERIALES Y MÉTODOS}

\section{COLECTA Y CONSERVACIÓN DE MATERIAL BIOLÓGICO}

La colecta de muestras se realizó en el lago Imiría (Figura 1), ubicada al sur este de la ciudad de Pucallpa, en el distrito de Masisea, Provincia de Coronel Portillo, Región Ucayali (coordenadas geográficas 7358'; 74²8'W y 0831';0911'S). Para el estudio se colectaron 400 ejemplares. Cada espécimen fue fotodocumentado, luego de cada uno de ellos se colectó un fragmento de aleta (de aproximadamente 2 $\mathrm{cm}^{2}$ ) que fue conservado en alcohol al $96^{\circ}$ hasta su procesamiento.

\section{ANÁLISIS GENÉTICO}

La extracción del ADN se realizó de acuerdo al protocolo con CTAB de Doyle \& Doyle (1987). La caracterización genética fue realizada mediante el análisis de seis primers microsatélites diseñados por Farias et al., 2003 (Tabla 1): La reacción fue realizada en un volumen total de $10 \mu \mathrm{l}$ conteniendo $0.5 \mu \mathrm{l}$ de ADN molde (100ng/ul) $5 \mathrm{U} / \mu \mathrm{l}$ de Taq polimerasa, $10 \mathrm{X}$ de Buffer, $25 \mathrm{mM}$ de $\mathrm{MgCl}_{2}, 10 \mathrm{mM}$ de dNTPs, $10 \mu \mathrm{M}$ de cebadores y agua ultrapura. Las condiciones de temperatura fueron: un ciclo de denaturación inicial a $95^{\circ} \mathrm{C}$ x $3 \mathrm{~min}$, seguido de 28 ciclos consistente en una denaturación $\left(95^{\circ} \mathrm{C} \mathrm{x} 40\right.$ seg. $)$, hibridación x 1 min con temperatura óptima para cada cebador y extensión $\left(72^{\circ} \mathrm{C}\right.$ x $\left.1 \mathrm{~min}\right)$; seguido de un ciclo de extensión final a $72^{\circ} \mathrm{C}$ x $30 \mathrm{~min}$. Los segmentos amplificados fueron denaturados y separados mediante electroforesis capilar utilizando un secuenciador (Applied Biosystems) 3130XL. Los pesos de los alelos de cada microsatélite fueron visualizados usando el software Peak Scanner versión 1.0 (https://products.appliedbio systems.com). 
La estructuración genética de especimenes estudiados fue determinado mediante el Análisis Factorial de Correspondencia (AFC) con auxilio del software Genetix versión 4.05.2 (Belkhir et al., 2004). La diversidad genética evaluada con los estadísticos descriptivos: numero de alelos por locus, heterocigosidad observada (Ho) y esperada (He), la diferencia a la panmixia (Fis), así como la frecuencia de alelos fue estimado con el software software Genetix versión 4.05.2 (Belkhir et al., 2004).

\section{RESULTADOS Y DISCUSIÓN}

La caracterización de los 400 ejemplares de paiche analizados mediante los seis loci microsatélites mostró un total de 68 alelos (Tabla 2). Observándose que los locus Agi12 y Agi10 fueron los mas polimorficos presentando el mayor número de alelos (siete y cinco alelos respectivamente). En tanto que el locus Agi09 presento un solo alelo, es decir fue monomórfico (invariable). La media de alelos por locus fue de $3.5( \pm 2.3)$. Las frecuencias alelicas variaron de 0.001 en los alelos 211 (loci Agi10) y 285 (loci Agi04), a 1 en el alelo 282 del locus Agi09, mostrando que los 400 individuos analizados presentan el mismo alelo en este locus.

Los valores de heterocigosidad esperada $(\mathrm{He})$ variaron de 0 en el locus Agi13 a 0.925 en el locus Agi12. En tanto que los valores de heterocigosidad observada (Ho) variaron de 0.002 en el locus Agi4 a 0.634 en el locus Agi10. La heterocigosidad multilocus observada fue diferente a la esperada, indicando una diferencia a la panmixia (reproducción al azar) en estos ejemplares, así como una fuerte deficiencia de heterocigotos (Tabla 2). Esto puede deberse a que los individuos introducidos no provienen de una población natural sino que son progenies obtenidas en la piscicultura a partir de un número reducido de reproductores. En este caso el efecto de bootleneck (cuello de botella) ha producido una reducción drástica de la diversidad genética de los peces cultivados en comparación de su población de origen.

Los 400 ejemplares de paiche analizados presentaron un total de 68 perfiles genéticos o genotipos (Tabla 3). La distribución de los genotipos entre los ejemplares es desproporcional, observándose que dos de estos perfiles genéticos (genotipos AW y AX) es compartido por 153 individuos ( 82 y 71 respectivamente); en tanto que 36 individuos presentan genotipos propios, es decir no comparten su perfil genético con ningún otro individuo. Si analizamos la distribución de genotipos en términos de porcentualidad (Figura 2) encontramos que un elevado numero de individuos comparten solo nueve genotipos (mientras 38\% de individuos comparte solo dos perfiles genéticos (genotipos AW y
AX), un $9 \%$ presentan genotipos únicos (36 individuos $=36$ genotipos). Este elevado nivel de homogeneidad encontrado entre el 38\% los ejemplares de paiche es probablemente un indicativo del alto nivel de parentesco entre estos individuos, es decir que probablemente desciende de un grupo reducido de reproductores.

Los resultados del análisis Factorial de correspondencia (AFC) corroboran la existencia de una fuerte semejanza genética entre la mayoría de los 400 ejemplares de paiche analizados (figura 3 ). La proyección grafica de los individuos muestra dos principales agrupaciones, la primera (Grupo A) conformada por 62 genotipos que reúnen 393 ejemplares (98.25\%), y la segunda (grupo B) por seis genotipos que reúne solo siete ejemplares $(1.75 \%)$ claramente diferenciados del primer grupo, que podrían representar individuos de un lote diferente de reproductores (provenientes de otra cuenca hidrográfica o en su defecto ejemplares nativos del lago Imiria). La distancia genética entre el primer y segundo grupo fue de 0.298, indicando una diferenciación genética marcada entre ellos. El cruzamiento de individuos genéticamente distintos a aquellos encontrados en una población natural puede promover la perdida de genes importantes de adaptabilidad al ambiente, que puede influenciar en la supervivencia de progenies en el ambiente natural (Lopera et al., 2008).

Dentro de la primera agrupación podemos comprobar que el $60.25 \%$ de ejemplares (241 individuos) que se encuentran reunidos en 13 de los 68 genotipos se encuentran muy relacionados entre si (circulo con línea discontinua en el grupo A de la figura 3), demostrando la gran cercanía genética entre la mayoría de individuos. En la naturaleza poblaciones con reducida diversidad genética con el paso del tiempo tienden a incrementar la taza de perdida de diversidad genética por efecto de la deriva génica. Lo que es agravado por el apareamiento entre parientes, que puede determinar la aparición de fenómenos de depresión consanguínea. La viabilidad genética de los ejemplares de Paiche introducidos en el lago Imiria se ve seriamente comprometida, ya que la variabilidad genética representa el seguro adaptativo frente a la impredicibilidad ambiental. Una perdida de variabilidad corresponde a una disminución de la eficacia biológica como consecuencia de una mayor susceptibilidad a enfermedades de las poblaciones (Frankham, 1995). En el caso de los ejemplares de paiche utilizados en el repoblamiento del lago Imiría si bien el número de perfiles genéticos no es bajo, pero existe una marcada desproporción en el número de individuos dentro de cada genotipo. Pudiéndose observar que un número reducido de genotipos 
engloba a más del $50 \%$ de los ejemplares utilizados en este repoblamiento. Además un numero bajo de reproductores por efecto de consanguinidad va inducir una disminución de la variabilidad genética (Aho et al., 2006). Esos problemas pueden consecuentemente afectar las poblaciones naturales de peces (Sønstebø et al., 2007) y el ecosistema en general, pudiendo conducir la especie a la extinción en esa área (Agostinho et al., 2005).

Por lo que se recomienda que en futuros eventos de repoblamiento se lleve en consideración la estructuración genética (diferenciación genética) reportada para la especie por García-Dávila et al. (2011), ha fin de evitar la introducción de stocks foráneos que puedan afectar seriamente las poblaciones nativas. Principalmente porque nuevos tipos de cruzamientos que causa rompimiento de los complejos genéticos que permiten la adaptación a las peculiaridades del medio, o introducción de parásitos y enfermedades con los que no han tenido contacto previo las poblaciones nativas (Hindar et al., 1991;
Ryman \& Laikre, 1991). Es decir que los futuros eventos de repoblamiento de paiche en el lago Imiria consideren el repoblamiento con ejemplares genéticamente lo más cercano posible de la población nativa del lago (ejemplares extraídos de cuerpos de agua cercanos al lago Imiría). También se recomienda que se considere el genotipado de los ejemplares a utilizar en los programas de repoblamiento para asegurar la introducción de nuevos genotipos que permitan aumentar la diversidad genética del paiche en este lago, que garantizara la sobrevivencia de la especie a través del tiempo en esta área.

Por ultimo, se recomienda también, que los futuros programas de repoblamiento con paiche, se apliquen en aquellas áreas realmente deterioradas y dentro de un modelo de gestión autosostenible. Igualmente, se recomienda evaluar la cantidad de individuos a liberar en relación con el tamaño efectivo poblacional y el grado de diferenciación de las poblaciones repobladas para evitar cambios genéticos en dichas poblaciones.

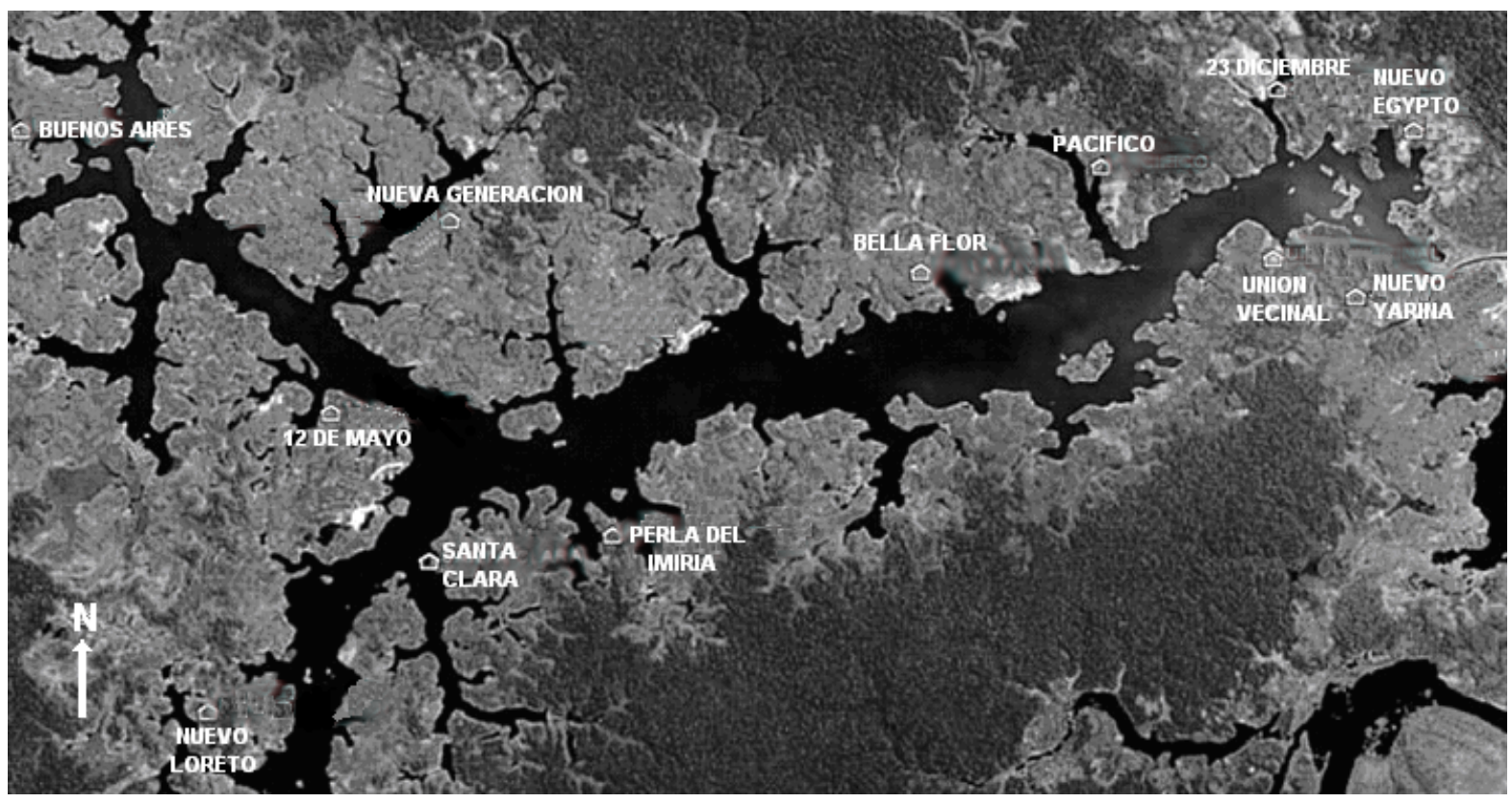

Figura 1. Imagen satelital del lago Imiría, donde fueron liberadas los especimenes de paiche Arapaima gigas evaluadas en este estudio. 


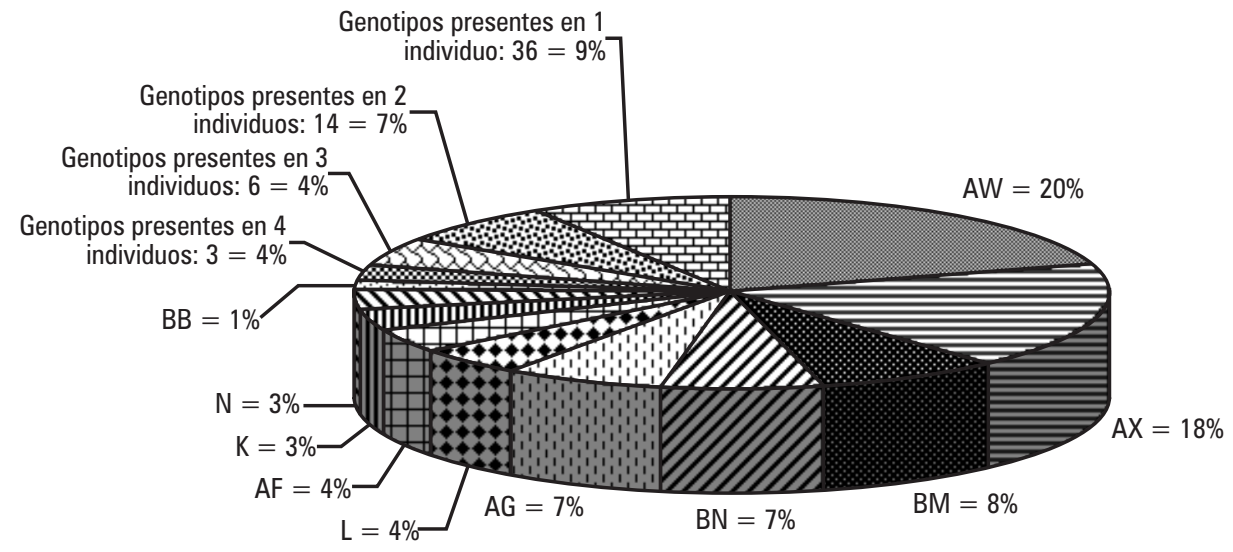

Figura 2. Composición porcentual de los principales genotipos encontrados en los 400 ejemplares de paiche Arapaima gigas utilizados en el programa de repoblamiento del lago Imiria.

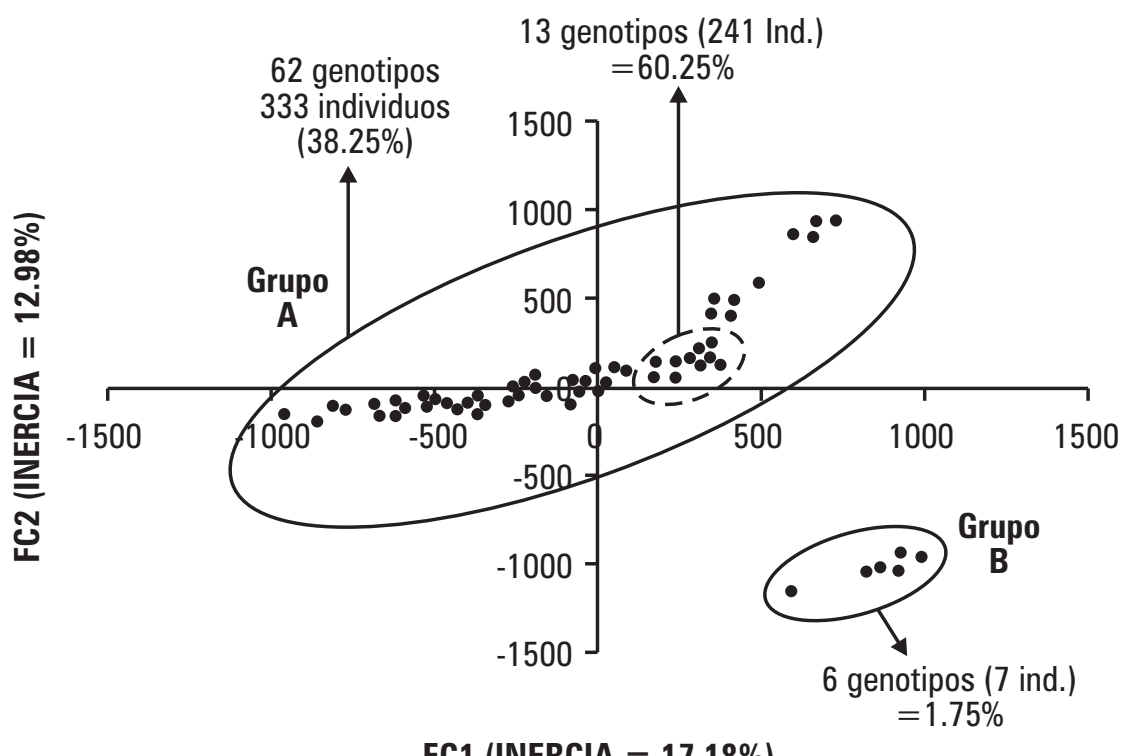

Figura 3. Proyección grafica de los resultados de AFC para los ejes 1 y 2 encontrados en 400 ejemplares de paiche del programa de repoblamiento del lago Imiria. 
FOLIA

Tabla 1. Nombre, motivo de repetición, secuencia, temperatura de anelamiento y rango de tamaño de alelos de los seis primers microsatélites utilizados para el genotipado de ejemplares de paiche Arapaima gigas analizadas.

\begin{tabular}{cllcc}
\hline NOMBRE & \multicolumn{1}{c}{$\begin{array}{c}\text { MOTIVO DE } \\
\text { REPETICIÓN }\end{array}$} & \multicolumn{1}{c}{ SECUENCIA } & $\begin{array}{c}\text { T}^{0} \\
\text { ANELAMIENTO }\end{array}$ & $\begin{array}{c}\text { RANGO DE } \\
\text { TAMAÑN ALELOS }\end{array}$ \\
\hline Agi04 & $(\mathrm{CT})_{29}$ & $\begin{array}{l}\text { FNET: GCAGCCCCAGTCTTGGAAGG } \\
\text { R: AGCACGGTGAATATCTGTGC }\end{array}$ & 62 & $258-280$ \\
Agi07 & $(\mathrm{CA})_{22}$ & $\begin{array}{l}\text { FFAN: AGCTCTCAGTACTGATGCTG } \\
\text { R: CCGATCATCTGTTTGCTCTG }\end{array}$ & 62 & $295-323$ \\
Agi09 & $(\mathrm{GTA})_{2}(\mathrm{CA})_{27}$ & $\begin{array}{l}\text { FHEX: TGAAAATCCTGTGGGACCTG } \\
\text { R: CCTAAAAACACATCACACTG }\end{array}$ & 58 & $300-334$ \\
Agi10 & $(\mathrm{CA})_{19}$ & $\begin{array}{l}\text { FNET: GGCCTACATCAAGCACTTAA } \\
\text { R: TTACTGGGTTGAGTTTGAC }\end{array}$ & 58 & $226-244$ \\
Agi12 & $(\mathrm{GA})_{2}(\mathrm{CA})_{9} \mathrm{CTCACT}(\mathrm{CA})_{20}$ & $\begin{array}{l}\text { FFAM: TTACTGAGGGCAATGACACG } \\
\text { R: TGATTACTCAGCAGGTCCTG }\end{array}$ & 58 & $357-375$ \\
Agi13 & $(\mathrm{CA})_{12}$ & $\begin{array}{l}\text { FHEX: GGAATGAGCAGGTTCCCAG } \\
\text { R: CTCCCTCTTCTGACATGACG }\end{array}$ & 62 & $263-267$ \\
\hline
\end{tabular}

Tabla 2. Composición de alelos, frecuencias, heterosigosidad esperada, heterocigosidad observada, encontrados en los seis loci microsatelites de paiche Arapaima gigas analizados.

\begin{tabular}{|c|c|c|c|c|c|c|c|c|}
\hline LOCUS & ALELOS & FRECUENCIA & LOCUS & ALELOS & FRECUENCIA & LOCUS & ALELOS & FRECUENCIA \\
\hline \multirow[t]{10}{*}{ Agi12 } & 317 & 0.009 & Agi10 & 211 & 0.001 & Agi07 & 295 & 0.069 \\
\hline & 319 & 0.424 & & 229 & 0.177 & & 309 & 0.187 \\
\hline & 321 & 0.003 & & 231 & 0.397 & & 319 & 0.714 \\
\hline & 327 & 0.066 & & 245 & 0.004 & & 323 & 0.030 \\
\hline & 329 & 0.014 & & 247 & 0.421 & & & \\
\hline & 331 & 0.427 & & & & & & \\
\hline & 333 & 0.058 & & & & & & \\
\hline & $\mathrm{H}_{0}$ & 0.631 & & & 0.634 & & & 0.449 \\
\hline & $\mathrm{H}_{\mathrm{e}}$ & 0.925 & & & 0.545 & & & 0.462 \\
\hline & $\mathrm{F}_{\text {is }}$ & $-0.141^{* * *}$ & & & $0.141^{* * *}$ & & & -0.030 \\
\hline Locus & Alelos & Frecuencia & Locus & Alelos & Frecuencia & Locus & Alelos & Frecuencia \\
\hline \multirow[t]{5}{*}{ Agi13 } & 268 & 0.023 & Agi04 & 279 & 0.999 & Agi09 & 282 & 1.000 \\
\hline & 270 & 0.977 & & 285 & 0.001 & & & \\
\hline & $\mathrm{H}_{0}$ & 0.049 & & & 0.002 & & & - \\
\hline & $\mathrm{H}_{\mathrm{e}}$ & 0.000 & & & 0.002 & & & - \\
\hline & $\mathrm{F}_{\text {is }}$ & $1.000 * * *$ & & & $-0.000 * * *$ & & & - \\
\hline \multirow[t]{3}{*}{ Multilocus } & $\mathrm{H}_{0}$ & 0.294 & & & & & & \\
\hline & $\mathrm{H}_{\mathrm{e}}$ & 0.323 & & & & & & \\
\hline & $\mathrm{F}_{\text {is }}$ & -0.096 & & & & & & \\
\hline
\end{tabular}


Tabla 3. Resumen de la composición de perfiles genéticos encontrados en los 400 especimenes de paiche Arapaima gigas utilizados en el programa de repoblamiento del lago Imiria.

\begin{tabular}{|c|c|c|c|c|c|c|c|c|}
\hline \multirow{2}{*}{$\mathrm{N}^{0}$} & \multicolumn{6}{|c|}{ LOCUS } & \multirow{2}{*}{ GENOTIPO } & \multirow{2}{*}{$\begin{array}{c}N^{0} \text { DE } \\
\text { EJEMPLARES }\end{array}$} \\
\hline & Agi10 & Agi09 & Agi12 & Agi13 & Agi04 & Agi07 & & \\
\hline 1 & 211231 & 282282 & 319331 & 270270 & 279279 & 309319 & $A$ & 1 \\
\hline 2 & 229229 & 282282 & 319331 & 270270 & 279279 & 295295 & C & 1 \\
\hline 3 & 229229 & 282282 & 319331 & 270270 & 279279 & 309319 & D & 1 \\
\hline 4 & 229229 & 282282 & 319331 & 270270 & 279279 & 295319 & $\mathrm{E}$ & 2 \\
\hline 5 & 229229 & 282282 & 319331 & 270270 & 279279 & 319319 & $\mathrm{~F}$ & 3 \\
\hline 6 & 229229 & 282282 & 319333 & 270270 & 279279 & 295323 & G & 3 \\
\hline 7 & 229229 & 282282 & 319333 & 270270 & 279279 & 295295 & H & 2 \\
\hline 8 & 229229 & 282282 & 319333 & 270270 & 279279 & 295319 & I & 2 \\
\hline 9 & 229229 & 282282 & 319333 & 270270 & 279279 & 319323 & $J$ & 1 \\
\hline 10 & 229229 & 282282 & 327327 & 270270 & 279279 & 319319 & K & 14 \\
\hline 11 & 229229 & 282282 & 327331 & 270270 & 279279 & 319319 & L & 18 \\
\hline 12 & 229229 & 282282 & 327331 & 270270 & 279279 & 319323 & $M$ & 1 \\
\hline 13 & 229229 & 282282 & 331331 & 270270 & 279279 & 319319 & $\mathrm{~N}$ & 13 \\
\hline 14 & 229231 & 282282 & 319331 & 270270 & 279279 & 309319 & $\tilde{N}$ & 1 \\
\hline 15 & 229231 & 282282 & 319333 & 270270 & 279279 & 295323 & 0 & 1 \\
\hline 16 & 229247 & 282282 & 319331 & 270270 & 279279 & 319319 & $P$ & 1 \\
\hline 17 & 229247 & 282282 & 319331 & 270270 & 279279 & 309319 & 0 & 1 \\
\hline 18 & 229247 & 282282 & 319331 & 270270 & 279279 & 295295 & $\mathrm{R}$ & 1 \\
\hline 19 & 229247 & 282282 & 319331 & 270270 & 279279 & 319323 & $S$ & 1 \\
\hline 20 & 229247 & 282282 & 319331 & 270270 & 279279 & 319319 & $\mathrm{~T}$ & 2 \\
\hline 21 & 229247 & 282282 & 319333 & 270270 & 279279 & 295319 & U & 3 \\
\hline 22 & 229247 & 282282 & 319333 & 270270 & 279279 & 295323 & V & 3 \\
\hline 23 & 229247 & 282282 & 319333 & 270270 & 279279 & 319323 & W & 1 \\
\hline 24 & 229247 & 282282 & 319333 & 270270 & 279279 & 295295 & $X$ & 2 \\
\hline 25 & 229247 & 282282 & 331331 & 270270 & 279279 & 295323 & Z & 1 \\
\hline 26 & 231231 & 282282 & 317329 & 270270 & 279279 & 319319 & $A A$ & 1 \\
\hline 27 & 231231 & 282282 & 317329 & 270270 & 279279 & 309319 & $A B$ & 1 \\
\hline 28 & 231231 & 282282 & 319321 & 270270 & 279279 & 319319 & $A C$ & 2 \\
\hline 29 & 231231 & 282282 & 319329 & 270270 & 279279 & 319319 & $A D$ & 1 \\
\hline 30 & 231231 & 282282 & 319331 & 270270 & 279279 & 295319 & $\mathrm{AE}$ & 1 \\
\hline 31 & 231231 & 282282 & 319331 & 270270 & 279279 & 309319 & $\mathrm{AF}$ & 16 \\
\hline 32 & 231231 & 282282 & 319331 & 270270 & 279279 & 319319 & $A G$ & 28 \\
\hline 33 & 231231 & 282282 & 319331 & 268268 & 279279 & 319319 & $\mathrm{AH}$ & 2 \\
\hline 34 & 231231 & 282282 & 327331 & 270270 & 279279 & 309319 & $\mathrm{Al}$ & 1 \\
\hline 35 & 231231 & 282282 & 319333 & 270270 & 279279 & 295295 & AJ & 2 \\
\hline 36 & 231231 & 282282 & 319333 & 268268 & 279279 & 319323 & AK & 1 \\
\hline 37 & 231231 & 282282 & 319333 & 270270 & 279279 & 295323 & $\mathrm{AL}$ & 1 \\
\hline
\end{tabular}


FOLIA CARACTERIZACIÓN GENÉTICA DE EJEMPLARES DE PAICHE Arapaima gigas (Cuvier, 1829) Amazónica UTILIZADOS EN EL REPOBLAMIENTO DEL LAGO IMIRIA (CUENCA DEL RíO UCAYALI)

\begin{tabular}{|c|c|c|c|c|c|c|c|c|}
\hline \multirow{2}{*}{$\mathbf{N}^{0}$} & \multicolumn{6}{|c|}{ LOCUS } & \multirow{2}{*}{ GENOTIPO } & \multirow{2}{*}{$\begin{array}{c}\mathrm{N}^{0} \text { DE } \\
\text { EJEMPLARES }\end{array}$} \\
\hline & Agi10 & Agi09 & Agi12 & Agi13 & Agi04 & Agi07 & & \\
\hline 38 & 231231 & 282282 & 319333 & 270270 & 279279 & 319323 & AM & 1 \\
\hline 39 & 231231 & 282282 & 331333 & 270270 & 279285 & 319323 & AN & 1 \\
\hline 40 & 231245 & 282282 & 319331 & 270270 & 279279 & 309319 & AÑ & 1 \\
\hline 41 & 231247 & 282282 & 317331 & 270270 & 279279 & 309319 & $\mathrm{AO}$ & 1 \\
\hline 42 & 231247 & 282282 & 317329 & 270270 & 279279 & 309319 & AP & 2 \\
\hline 43 & 231247 & 282282 & 317329 & 270270 & 279279 & 319319 & $\mathrm{AO}$ & 2 \\
\hline 44 & 231247 & 282282 & 319319 & 268268 & 279279 & 319319 & $A R$ & 1 \\
\hline 45 & 231247 & 282282 & 319329 & 270270 & 279279 & 309319 & AS & 1 \\
\hline 46 & 231247 & 282282 & 319329 & 270270 & 279279 & 319319 & AT & 2 \\
\hline 47 & 231247 & 282282 & 319331 & 270270 & 279279 & 295295 & $\mathrm{AU}$ & 4 \\
\hline 48 & 231247 & 282282 & 319331 & 270270 & 279279 & 295323 & $\mathrm{AV}$ & 1 \\
\hline 49 & 231247 & 282282 & 319331 & 270270 & 279279 & 309319 & AW & 82 \\
\hline 50 & 231247 & 282282 & 319331 & 268268 & 279279 & 309319 & $\mathrm{Y}$ & 1 \\
\hline 51 & 231247 & 282282 & 319331 & 270270 & 279279 & 319319 & $A X$ & 71 \\
\hline 52 & 231247 & 282282 & 319331 & 268268 & 279279 & 319319 & $A Y$ & 1 \\
\hline 53 & 231247 & 282282 & 319331 & 270270 & 279279 & 319323 & $A Z$ & 1 \\
\hline 54 & 231247 & 282282 & 319333 & 270270 & 279279 & 319323 & BA & 3 \\
\hline 55 & 231247 & 282282 & 319333 & 270270 & 279279 & 319319 & BB & 5 \\
\hline 56 & 231247 & 282282 & 319333 & 270270 & 279279 & 295295 & $B C$ & 4 \\
\hline 57 & 231247 & 282282 & 319333 & 270270 & 279279 & 295319 & $\mathrm{BD}$ & 4 \\
\hline 58 & 231247 & 282282 & 319333 & 270270 & 279279 & 295323 & $\mathrm{BE}$ & 1 \\
\hline 59 & 231247 & 282282 & 319333 & 270270 & 279279 & 309319 & $\mathrm{BF}$ & 2 \\
\hline 60 & 231247 & 282282 & 327331 & 270270 & 279279 & 309319 & $B G$ & 1 \\
\hline 61 & 231247 & 282282 & 327331 & 268268 & 279279 & 309319 & $\mathrm{BH}$ & 1 \\
\hline 62 & 231247 & 282282 & 327331 & 270270 & 279279 & 319319 & $\mathrm{BI}$ & 3 \\
\hline 63 & 231247 & 282282 & 331331 & 270270 & 279279 & 309319 & BJ & 1 \\
\hline 64 & 245247 & 282282 & 319331 & 270270 & 279279 & 319319 & BK & 2 \\
\hline 65 & 247247 & 282282 & 319329 & 270270 & 279279 & 309319 & $\mathrm{BL}$ & 1 \\
\hline 66 & 247247 & 282282 & 319331 & 270270 & 279279 & 309319 & BM & 32 \\
\hline 67 & 247247 & 282282 & 319331 & 270270 & 279279 & 319319 & BN & 28 \\
\hline 68 & 247247 & 282282 & 319331 & 270270 & 279279 & 319323 & BÑ & 1 \\
\hline
\end{tabular}




\section{AGRADECIMIENTO}

Al Gobierno Regional de Ucayali por el financiamiento de la presente investigación a través del proyecto "Preservación del paiche en la laguna Imiría, distrito de Masisea, provincia de Coronel Portillo"

\section{BIBLIOGRAFIA CITADA}

Agostinho, A.A.; Thomaz, S.M.; Gomes, L.C. 2005. Conservation of the biodiversity of Brazil's inland waters. Conserv. Biol., 19(3): 646-652.

Aho, T.; Rönn, J.; Piironen, J.; Björklund, M. 2006. Impacts of effective population size on genetic diversity in hatchery reared Brown trout (Salmo trutta L.) populations. Aquaculture, 253(1-4): 244248.

Belkhir, K.; Borsa, P.; Chichi, I.; Raufast, N.; Bonhomme, F. 2004. GENETIX 4.05.2. logiciel sous windowns TM pour la genétique des populations. Laboratoire génome. populations. interactions. CNRS UMR 5000. Université de Montpellier II. Montpellier. France.

Doyle, J.J.; Doyle, J.L. 1987. A rapid DNA isolation procedure for small quantities of fresh leaf tissue. Phytochem. Bull. 19:11-15.

Farias, I.; Hrbek, T.; Brinkmann,H.; Sampaio, I.; Meyer, A. 2003. Characterization and isolation of DNA microsatellite primers for Arapaima gigas. an economically important but severely overexploited fish species of the Amazon basin. Molecular Ecology Notes. 3. 128-130.

Frankham, R. 1995. Conservation genetics. Ann. Rev. Genet., 29: 305-327.

García-Dávila, C.; Castro-Ruiz, D.; Querouil, S.; Chota-Macuyama, W.; García, J.; Chu-Koo, F.; Duponchelle, F.; Núñez, J.; Renno, J.-F. 2011. Avances en el estudio de variabilidad genética de cinco poblaciones naturales de paiche Arapaima gigas en la Amazonía peruana. Resumen extendido del III simposio internacional "Biología de las poblaciones de peces de la Amazonía y piscicultura", Leticia - Colombia.

Goulding, M. 1980 Fishes and the Forest. University of California press. Los Angeles. CA.

Hindar, K.; Ryman, N.; Utter, F. 1991. Genetic effects of cultured fish on natural fish populations. Can.J. Fish. Aquat. Sci., 48: 945-957.

Lopera, N.M.; Pereira, R.; A. Povh, J.; Gomes, P.; Lauro Vargas, L.; Nogueira, S. 2008. Caracterización genética de lotes de peces usados en programas de repoblamiento y su importancia en la conservación genética en la piscicultura. Zootecnia Trop. 26(4): 515-522.

Menezes, R.S. 1951 Notas biológicas e econômicas sobre o pirarucu Arapaima gigas (Cuvier) (Actinopterygii. Arapaimidae). Serviço de Informação Agrícola / Ministerio da Agricultura. Serie estudos técnicos. 3: 9-39.

Nelson, J. S. 1994 Fishes of the World. 3rd edition. John Wiley and Sons. Inc.. New York. NY. 624 pp.

Peakall, R.; Smouse, P.E. 2005. GenAlEx V6: Genetic analysis in Excel. Population genetic software for teaching and research. Australian National University. Canberra. Disponible via http://www.anu.edu.au/BoZo/GenAlEx.

Ryman, N. 1981. Conservation of genetic resources: Experiences from the brown trout (Salmo trutta). Ecol. Bull., 34: 61-74.

Ryman, N.; Laikre, L. 1991. Effects of supportive breeding on the genetically effective population size. Conserv. Biol., 5: 325-329.

Sønstebø J.H., Borgstrøm, R.; Heun, M. 2007. Genetic structure of brown trout (Salmo trutta L.) from the Hardangervidda mountain plateau (Norway) analyzed by microsatellite DNA: a basis for conservation guidelines. Conserv. Genet., 8(1): 33-44.

Veríssimo, J. 1895 A Pesca na Amazônia. Livraria Clássica. de Alves \& C.. Rio de Janeiro. Brazil. 130 pp. 